\title{
Hamilton paths in Cayley graphs on generalized dihedral groups
}

\author{
Brian Alspach \\ School of Mathematical and Physical Sciences, University of Newcastle \\ Callaghan, NSW 2308, Australia \\ C. C. Chen \\ Integrated Decision Systems Consultancy Pte Ltd, 06-06 \\ Pacific Tech Centre, Singapore 159303 \\ Matthew Dean \\ Mathematics Department, The University of Queensland \\ St Lucia, Queensland 4072, Australia
}

Received 17 March 2009, accepted 25 January 2010, published online 1 February 2010

\begin{abstract}
We investigate the existence of Hamilton paths in connected Cayley graphs on generalized dihedral groups. In particular, we show that a connected Cayley graph of valency at least three on a generalized dihedral group, whose order is divisible by four, is Hamiltonconnected, unless it is bipartite, in which case it is Hamilton-laceable.
\end{abstract}

Keywords: Hamilton-connected, Hamilton-laceable, Cayley graph, generalized dihedral group, honeycomb toroidal graph

Math. Subj. Class.: 05C25, 05C70

\section{Introduction}

A family of trivalent vertex-transitive graphs that have garnered attention over the last thirty-five years have been called brick products in [1, 2, 5], honeycomb tori in [8, 9, 12, 13, $14,15]$, honeycomb toroidal graphs in [3], and hexagonal toroidal embeddings in [6, 11]. Altshuler [6] studied them when he was considering Hamilton cycles in graphs embedded

E-mail addresses: brian.alspach@newcastle.edu.au (Brian Alspach), ccchen@idsc.com.sg (C. C. Chen), mdean@uq.edu.au (Matthew Dean) 
in the torus. He was unable to prove that all of them are hamiltonian. That all are hamiltonian was first proven in [5] and independently proven in [15]. Hamilton paths in these graphs were considered in [1, 2].

Recently [3] it was shown that these trivalent graphs are Cayley graphs on generalized dihedral groups. In this paper we study Hamilton paths in the latter family of graphs. The results obtained, of course, then apply to the subfamily of honeycomb toroidal graphs mentioned above.

We now give some definitions to set the stage for the development of the material.

Definition 1.1. Let $G$ denote a finite group and let $S$ be a subset of $G$ satisfying

1. $1 \notin S$, where 1 denotes the identity of $G$, and

2. $s \in S$ implies $s^{-1} \in S$.

The Cayley graph $\operatorname{Cay}(G ; S)$ on $G$ with connection set $S$ has the elements of $G$ as its vertices and an edge joining $g$ to $g s$ for all $g \in G$ and $s \in S$.

We emphasize that we consider only finite groups and finite graphs in this paper. There are two families of Cayley graphs we consider. They arise from the family of dihedral groups and the family of generalized dihedral groups. Even though the former is a subfamily of the latter, we distinguish the dihedral groups because they have such a rich history on their own.

Definition 1.2. The dihedral group $D_{n}$ is the group of order $2 n$ with generators $\rho$ and $\tau$ that satisfy

$$
\rho^{n}=\tau^{2}=1 \text { and } \tau \rho \tau=\rho^{-1} .
$$

Definition 1.3. Let $H$ be a finite abelian group. The generalized dihedral group $D_{H}$ is the group of order $2|H|$ generated by $H$ and $\tau$ where $\tau \notin H, \tau^{2}=1$, and $\tau h \tau=h^{-1}$ for all $h \in H$.

Definition 1.4. The honeycomb toroidal graph $\operatorname{HTG}(m, 2 n, s)$, where $m+s$ is even, $m \geqq$ 1 , and $n \geqq 2$, is the trivalent graph defined as follows. It has vertex set $\left\{u_{i, j}: 0 \leqq i \leqq\right.$ $m-1,0 \leqq j \leqq 2 n-1\}$, and it has the following edges where all the arithmetic is carried out on the subscripts modulo $m$ or $2 n$ as appropriate. For each $i \in\{0,1, \ldots, m-1\}, u_{i, j}$ $i$ adjacent to $u_{i, j-1}$ and $u_{i, j+1}$. For each even $i \in\{0,1, \ldots, m-2\}$, there is an edge from $u_{i, j}$ to $u_{i+1, j}$ for all odd $j$. For each odd $i \in\{0,1, \ldots, m-2\}$, there is an edge from $u_{i, j}$ to $u_{i+1, j}$ for all even $j$. If $m-1$ is even, there is an edge from $u_{m-1, j}$ to $u_{0, j+s}$ for all odd $j$. If $m-1$ is odd, there is an edge from $u_{m-1, j}$ to $u_{0, j+s}$ for all even $j$.

We are not using the terminology generalized honeycomb tori because a torus is a surface and even though the graphs embed on the torus, we think it better to not treat the graph itself as a surface. Instead, we use the terminology honeycomb toroidal graphs. There is some nomenclature we use throughout this paper for honeycomb toroidal graphs that should be mentioned now. Edges that belong to the $2 n$-cycles induced on the vertices $u_{i, j}$, with $i$ fixed, are called vertical edges. Edges of the form $u_{i, j} u_{i+1, j}$, where $0 \leqq i \leqq m-2$, are called flat edges. Edges joining vertices of the column indexed 0 and the column indexed $m-1$ are called jump edges. The latter edges are said to have jump s.

The following result was proven in [3]. 
Theorem 1.5. The honeycomb toroidal graph $\operatorname{HTG}(m, 2 n, s)$ is a Cayley graph on the generalized dihedral group $D_{H}$, where $H$ is either a cyclic group or a direct product of two cyclic groups.

Recall that a graph $X$ is Hamilton-connected if, for every pair of vertices $u, v \in V(X)$, there is a Hamilton path whose terminal vertices are $u$ and $v$. Similarly, a bipartite graph with parts $A$ and $B$ is Hamilton-laceable if, for all vertices $u \in A$ and $v \in B$, there is a Hamilton path whose terminal vertices are $u$ and $v$. The latter, of course, forces $|A|=|B|$ to be the case.

Definition 1.6. We say that a family $\mathcal{F}$ of graphs is $H^{*}$-connected when every non-bipartite graph in $\mathcal{F}$ is Hamilton-connected and every bipartite graph in $\mathcal{F}$ is Hamilton-laceable.

The most celebrated theorem relating Cayley graphs and $H^{*}$-connectivity is the following theorem of Chen and Quimpo [7].

Theorem 1.7. The family of connected Cayley graphs of valency three or more on abelian groups is an $H^{*}$-connected family.

Theorem 1.7 has been extended to the family of connected Cayley graphs of valency three or more on hamiltonian groups [4]. In this paper we consider the extension to generalized dihedral groups. The main result is the following theorem.

Theorem 1.8. The family of connected Cayley graphs of valency at least 3 on generalized dihedral groups, whose orders are divisible by 4 , is an $H^{*}$-connected family.

Outline of proof. The proof of Theorem 1.8 is long, indeed, the rest of the paper, except two short corollaries at the end, consists of the proof. Consequently, a few words about the proof are in order. Given a Cayley graph $X=\operatorname{Cay}\left(D_{H} ; S\right)$ on the generalized dihedral group $D_{H}$, throughout the paper we let $S_{1}$ denote $S \cap H, S_{2}$ denote $S \cap H \tau, X_{1}$ denote the subgraph induced on $H$ by $X$, and $X_{2}$ denote the subgraph induced on $H \tau$ by $X$.

Of course, $X_{1}$ and $X_{2}$ are isomorphic Cayley graphs on abelian groups so that Theorem 1.7 may be applied for these subgraphs. It should be no surprise then that the further we are from being able to apply Theorem 1.7, the more work we must perform. Hence, the proof has three basic cases: $S_{1}=\emptyset,\left|S_{1}\right|=1$, and $\left|S_{1}\right| \geqq 2$.

There are several techniques that are used in the three cases and these are presented as separate lemmas in Section 2 in order to improve the exposition for the three cases and to avoid repetition for those techniques which are applied more than once. Section 3 addresses honeycomb toroidal graphs. They form the basis for establishing Theorem 1.8 when $S_{1}=\emptyset$.

Section 4 then contains the proof of the theorem. The case $S=\emptyset$ is completed via induction on $\left|S_{2}\right|$. The case $\left|S_{1}\right|=1$ takes some work too but not as much as the preceding case. The case $\left|S_{1}\right| \geqq 2$ is fairly easy because of the power of Theorem 1.7 and techniques developed in Section 2.

\section{Basic toolkit}

We now present some useful lemmas as described above. 
Definition 2.1. Let $P=u_{0} u_{1} \cdots u_{k}$ be a path of length $k$ in some graph. If $u_{k}$ is adjacent to a vertex $u_{r}$ on $P$, where $u_{r} \neq u_{k-1}$, then deleting the edge $u_{r} u_{r+1}$ from $P$ and inserting the edge joining $u_{r}$ to $u_{k}$ is called a Posa exchange. We say that we have performed a Posa exchange on $P$ using the edge $u_{k} u_{r}$. Note that we obtain a path from $u_{0}$ to $u_{r+1}$ spanning the same set of vertices as $P$. In particular, when $P$ is a Hamilton path, we obtain another Hamilton path with one end vertex altered.

There is a structural property of the Cayley graphs under discussion that is used frequently. It is embodied in the next lemma, but before embarking on the proof, there are some useful observations to be made.

Given a Cayley graph on some group $G$, left multiplication by a group element from $G$ is a graph automorphism. From this we see that the subgraphs induced by a Cayley graph on distinct left cosets of a subgroup of $G$ are isomorphic. Thus, in general, for subgraph properties in Cayley graphs it is more convenient to think in terms of left cosets when a subgroup is involved. On the other hand, given a vertex in a Cayley graph its neighbors are determined by right multiplication. So getting from one vertex to another is more conveniently thought of in terms of right multiplication. This makes right cosets also useful when thinking about various properties of Cayley graphs.

Lemma 2.2. Let $X$ be a Cayley graph on a generalized dihedral group $D_{H}, H^{\prime}$ be a subgroup of $H$, and $Y_{1}$ and $Y_{2}$ be the subgraphs induced by $X$ on two distinct cosets of $H^{\prime}$. If there is an edge from the vertex $g_{1} \in Y_{1}$ to $g_{1} s \in Y_{2}$, then the element $s \in S$ generates $a$ perfect matching between $Y_{1}$ and $Y_{2}$, that is, for every $g \in Y_{1}$ the vertex $g s \in Y_{2}$.

Proof. The subgroup $H^{\prime}$ is normal in $D_{H}$ so that $g H^{\prime}=H^{\prime} g$ for all $g \in D_{H}$, that is, left and right cosets of $H^{\prime}$ are the same. That is why the statement of the theorem only refers to cosets of $H^{\prime}$. Let $Y_{1}^{\prime}$ be the subgraph induced on $H^{\prime}$ by $X$.

Consider a vertex labelled $h^{\prime}$ in $Y_{1}^{\prime}$. Consider any element $s$ in the connection set not belonging to $H^{\prime}$. Then the vertex labelled $h^{\prime}$ is adjacent to the vertex labelled $h^{\prime} s$ which belongs to the coset $H^{\prime} s$. Thus, if we multiply every element of $H^{\prime}$ on the right by $s$, we obtain $H^{\prime} s$. That is, the element $s$ in the connection set generates a perfect matching between the vertices labelled with elements of $H^{\prime}$ and those labelled with elements of $H^{\prime} s$. The result follows because left multiplication is a graph automorphism.

Lemma 2.3. Let $X$ be a bipartite graph containing vertex-disjoint bipartite subgraphs $Y_{0}$, $Y_{1}, \ldots, Y_{t}$, each of order at least 4 , with respective bipartitions $\left(A_{0}, B_{0}\right),\left(A_{1}, B_{1}\right), \ldots$, $\left(A_{t}, B_{t}\right)$ and which together span $X$. If each of the bipartite subgraphs is Hamiltonlaceable and there is a perfect matching joining the vertices of $A_{i}$ to the vertices of $B_{i+1}$ for $i=0,1, \ldots, t$, where we take $B_{t+1}$ to be $B_{0}$, then $X$ is Hamilton-laceable.

Proof. Choose an arbitrary vertex $u \in A_{0}$. We want to show there is a Hamilton path from $u$ to any vertex $v$ belonging to some $B_{j}$. First let $v \in B_{0}$. By hypothesis there is a Hamilton path $P_{0}$ in $Y_{0}$ from $u$ to $v$. Let $u v_{0}$ be the first edge of $P_{0}$. Remove this edge from $P_{0}$ and build a new path starting at $u$ by using the perfect matching edge from $u$ to $v_{1} \in B_{1}$. Then add a Hamilton path $P_{1}$ in $Y_{1}$ from $v_{1}$ to some $u_{1} \in A_{1}$. Extend this path by adding the matching edge from $u_{1}$ to $v_{2} \in B_{2}$. Now extend by adding a Hamilton path in $Y_{2}$ from $v_{2}$ to some $u_{2} \in A_{2}$. Continue in this way until reaching a vertex $v_{t} \in B_{t}$. Let $u_{t} \in A_{t}$ be the neighbor of $v_{0}$ in the perfect matching between $A_{t}$ and $B_{0}$. Extend the path by adding a Hamilton path from $v_{t}$ to $u_{t}$ in $Y_{t}$. Now add the edge from $u_{t}$ to $v_{0}$. We have replaced 
the edge $u v_{0}$ by a path that uses all vertices of $X$ not in $Y_{0}$. Thus, we have a Hamilton path in $X$ from $u$ to $v$.

If $v \in B_{1}$, then it is easy to find a Hamilton path from $u$ to $v$ by taking a Hamilton path in $Y_{0}$ from $u$ to some $v_{0} \in B_{0}$, then adding the matching edge into $A_{t}$, and then adding a Hamilton path using all the vertices in $Y_{t}$. Then move into $Y_{t-1}$ and continue around to $Y_{1}$. Because we enter $Y_{1}$ at a vertex of $A_{1}$, it is easy to complete to a Hamilton path from $u$ to $v$.

Let $v \in B_{i}$ with $i \notin\{0,1\}$. First choose a Hamilton path $P^{\prime}$ in $Y_{0}$ from $u$ to some $v_{0,0} \in B_{0}$. Let $v_{0,1} u_{0} v_{0,0}$ be the last two edges of $P^{\prime}$. Let $P$ be the subpath of $P^{\prime}$ from $u$ to $v_{0,1}$ and let $Q$ be the path consisting of the edge $u_{0} v_{0,0}$. Note that $P$ and $Q$ use all the vertices of $Y_{0}$.

We extend $Q$ first. Add the matching edge from $u_{0}$ to its neighbor $v_{1} \in B_{1}$. If $v \in B_{2}$, do not extend $Q$ any further in this direction at this time. If $v \notin B_{2}$, then let $u_{1} \in A_{1}$ and extend $Q$ by adding a Hamilton path in $X_{1}$ from $v_{1}$ to $u_{1}$. Continue extending $Q$ in this way so that the extension of $Q$ in this direction uses all vertices of $Y_{1}, Y_{2}, \ldots, Y_{i-2}$ and terminates with the matching edge from the last vertex of $Q$ in $A_{i-2}$ to a vertex $v_{i-1} \in$ $B_{i-1}$.

Extend both $P$ and $Q$ in the other direction as follows. Extend them into $Y_{t}$ by adding the matching edges from $v_{0,1}$ and $v_{0,0}$ to $u_{t, 1}$ and $u_{t, 0}$, respectively, in $A_{t}$. If $v \notin B_{t}$, then consider any Hamilton path in $Y_{t}$ starting with $u_{t, 0}$. Let $v_{t, 0}$ be the predecessor of $u_{t, 1}$ on this Hamilton path. Now extend $Q$ by adding the subpath from $u_{t, 0}$ to $v_{t, 0}$ and extend $P$ by adding the subpath from $u_{t, 1}$ to the terminal vertex $v_{t, 1}$. The extended paths use all the vertices of $Y_{t}$. Continue extending $P$ and $Q$ through $Y_{t-1}, Y_{t-2}$, and so on until completing $Y_{i+1}$.

Let the terminal vertices of the extended paths $Q$ and $P$ in $Y_{i+1}$ be $v_{i+1,0}$ and $v_{i+1,1}$, respectively. Add on the matching edges from both into $A_{i}$. Let the terminal vertices now be $u_{i, 0}$ and $u_{i, 1}$, respectively. We now describe how to combine everything into a single Hamilton path from $u$ to $v$. This also works when $v \in B_{t}$.

Let $R$ be a Hamilton path in $Y_{i}$ from $u_{i, 1}$ to $v$. As we traverse $R$ from $u_{i, 1}$ to $v$, let $v^{\prime}$ be the predecessor of $u_{i, 0}$. Now add the subpath of $R$ from $u_{i, 0}$ to $v$ to $Q$, and the subpath from $u_{i, 1}$ to $v^{\prime}$ to $P$. Let $u_{i-1}$ be the vertex of $A_{i-1}$ adjacent to $v^{\prime}$ by a matching edge. To the other end of $Q$ add a Hamilton path in $Y_{i-1}$ from $v_{i-1}$ to $u_{i-1}$, and the matching edge to $v^{\prime}$. The resulting path is a Hamilton path in $X$ joining $u$ and $v$. Because the bipartite subgraphs are joined in a cyclic fashion, the preceding argument works for any $u$ and $v$ in different parts of the bipartition of $X$.

Lemma 2.4. Let $X$ be a vertex-transitive graph containing a spanning subgraph $Y$ that consists of a perfect matching of t edges $e_{0}, e_{1}, \ldots, e_{t-1}$ such that $e_{i}$ is joined to $e_{i+1}$ by a 2-matching for $i=0,1, \ldots, t-1$, where $e_{t}=e_{0}$. If $X$ is bipartite, then $X$ is Hamiltonlaceable, and if $X$ is not bipartite, then $X$ is Hamilton-connected.

Proof. The edges $e_{0}, e_{1}, \ldots, e_{t-1}$ are joined by successive 2-matchings and we may assume the end vertices of $e_{i}$ are labelled with $u_{i}$ and $v_{i}$, respectively, so that $u_{i}$ is adjacent to $u_{i+1}$ and $v_{i}$ is adjacent to $v_{i+1}$ for $i=0,1, \ldots, t-2$. The key to the proof is the 2-matching between $e_{t-1}$ and $e_{0}$.

Let $Y$ be the spanning subgraph made up of the edges $e_{0}, e_{1}, \ldots, e_{t-1}$ and the 2matchings joining $e_{i}$ to $e_{i+1}, i=0,1, \ldots, t-1$. If $u_{0} u_{t}$ and $v_{0} v_{t}$ are edges of $Y$, then $Y$ is isomorphic to the cartesian product of a $t$-cycle and $K_{2}$. On the other hand, if $u_{0} v_{t}$ and 
$v_{0} u_{t}$ are edges of $Y$, then $Y$ is isomorphic to the circulant graph of order $2 t$ with connection set $\{ \pm 1, t\}$. Both possible spanning graphs are Cayley graphs on abelian groups so that $X$ is Hamilton-laceable by Theorem 1.7.

Moreover, when $Y$ is isomorphic to the cartesian product of a $t$-cycle and $K_{2}, Y$ is not bipartite if and only if $t$ is odd. When $Y$ is isomorphic to the circulant graph of order $2 t$ with connection set $\{ \pm 1, t\}, Y$ is not bipartite if and only if $t$ is even. Whenever $Y$ is not bipartite, it is Hamilton-connected by Theorem 1.7. This implies $X$ itself is Hamiltonconnected.

The only situation not covered is when $Y$ is bipartite but $X$ itself is not bipartite. The bipartition $(A, B)$ of $V(Y)$ is uniquely determined because $Y$ is connected. There must be an edge of $X$ joining two vertices in the same part because $X$ is not bipartite. Because of the cyclic labelling, we may assume there is an edge joining $u_{0}$ to either $u_{i}, i$ even, or $v_{j}$, $j$ odd.

Suppose there is an edge from $u_{0}$ to $u_{i}$ with $i$ even. Consider $u_{k}$, where $k$ is odd and $k<i$. Start a path

$$
u_{1} u_{2} \cdots u_{k-1} v_{k-1} v_{k-2} \cdots v_{1} v_{0} u_{0} .
$$

Then add the edge from $u_{0}$ to $u_{i}$ and continue to $u_{t-1}$ using all vertices $u_{i}, u_{i+1}, \ldots, u_{t-1}$. Now add the edge to $v_{t-1}$ and work back down from $v_{t-1}$ to $v_{i-1}$. Because both $k$ and $i-1$ are odd, there are an odd number of matching edges between $e_{k}$ and $e_{i-1}$, inclusive, so that it is easy to jump back and forth along successive matching edges until terminating at $u_{k}$.

In a similar manner it is easy to find Hamilton paths from $u_{1}$ to all other vertices in the same part as $u_{1}$. A similar argument works when $u_{0}$ is adjacent to some $v_{j}, j$ odd. Thus, given that there is an edge of $X$ between two vertices in the same part, then there exists a vertex that has Hamilton paths from it to any other vertex in $X$. This implies that $X$ is Hamilton-connected because it is vertex-transitive.

Lemma 2.5. Let $X$ be a graph and $Y$ be a spanning subgraph of $X$. Suppose $Y$ consists of vertex-disjoint subgraphs $Y_{0}, Y_{1}, \ldots, Y_{t}$ such that each of them is Hamilton-connected and has order at least 3, and they are joined together in one of the following two ways. Either

- $Y_{i}$ is joined to $Y_{i+1}$ by a perfect matching for $i=0,1, \ldots, t-1$, or

- each $Y_{i}$ has a partition $\left(A_{i}, B_{i}\right)$ such that $\left|A_{i}\right|=\left|B_{i}\right|$ and $A_{i}$ is joined by a perfect matching to $B_{i+1}$ for $i=0,1, \ldots, t$, where $B_{t+1}=B_{0}$.

\section{Then the graph $X$ is Hamilton-connected.}

Proof. First assume that $Y_{i}$ is joined to $Y_{i+1}$ by a perfect matching for $i=0,1, \ldots, t-1$. Let $u, v$ be two arbitrary vertices of $X$, where $u \in Y_{j}$ and $v \in Y_{k}$. Without loss of generality, we may assume that $j \leqq k$.

It is easy to find a path from $u$ to $v$ spanning the vertices of $Y_{j} \cup Y_{j+1} \cup \cdots \cup Y_{k}$. We do this by taking a path spanning $Y_{j}$ from $u$ and terminating at $v$ if $j=i$, or terminating at a $u^{\prime} \in Y_{j}$ such that the matching edge from $u^{\prime}$ to $Y_{j+1}$ is not incident with $v$. This is easy to do because there are at least three vertices in each $Y_{i}$. We then extend the path through $Y_{j+1}$ via the matching edge from $u^{\prime}$.

Once we have a path from $u$ to $v$ spanning the vertices of $Y_{j} \cup Y_{j+1} \cup \cdots \cup Y_{k}$, we extend it to a Hamilton path with $u$ and $v$ as terminal vertices by removing an edge in 
$Y_{j}$ and adding the two matching edges into $Y_{j-1}$ and then joining the new vertices with a path spanning $Y_{j-1}$. We may continue in this way until we have included all vertices of $Y_{i}$, $i<j$. We can include all the vertices from $Y_{i}$ with $i>k$ in the same way. We have shown that the graph $Y$ is Hamilton-connected because $u$ and $v$ are arbitrary.

Move to the case that $Y_{i}$ has a partition $\left(A_{i}, B_{i}\right)$ as described earlier, $i=0,1, \ldots, t$. Because of the way the subgraphs are joined, it suffices to find a Hamilton path from an arbitrary vertex $u \in A_{0}$ to any other vertex. So let $u$ be an arbitrary vertex of $A_{0}$. Let $v \in Y_{0}, v \neq u$, and let $P$ be a Hamilton path in $Y_{0}$ joining $u$ and $v$. There must be an edge $u_{0} v_{0}$ on $P$ such that as $P$ is traversed from $u$ to $v$, the edge is traversed from $u_{0}$ to $v_{0}$, and $u_{0} \in A_{0}$ and $v_{0} \in B_{0}$. Let $v_{1}$ be the vertex of $B_{1}$ adjacent to $u_{0}$ by a matching edge, and let $u_{t}$ be the vertex of $A_{t}$ adjacent to $v_{0}$ by a matching edge. Remove the edge $u_{0} v_{0}$ from $P$ and instead go from $u_{0}$ to $v_{1}$. Then extend that subpath by adding on a Hamilton path in $Y_{1}$ from $v_{1}$ to some $u_{1} \in A_{1}$. Then add on the matching edge from $u_{1}$ to $v_{2}$ in $B_{2}$. It is easy to see how we continue this until we reach $u_{t} \in A_{t}$. We then add on the matching edge to $v_{0}$ at which point we have a Hamilton path from $u$ to $v$.

If $v \in Y_{1}$, then we take a Hamilton path in $Y_{0}$ from $u$ to some $v_{0} \in B_{0}$. We then extend it by adding the matching edge from $v_{0}$ to a vertex in $A_{t}$. We then extend it by taking a Hamilton path in $Y_{t}$. We work back through successive $Y_{i}$ subgraphs until reaching $A_{1}$. We just make certain that we enter $A_{1}$ at a vertex different from $v$. We then add on a Hamilton path terminating at $v$.

If $v \in Y_{i}, i \notin\{0,1\}$, then take a Hamilton path in $Y_{0}$ from $u$ to some $u_{0} \in A_{0}$. Extend it by taking the matching edge from $u_{0}$ to some $v_{1} \in B_{1}$. Next extend it by taking a Hamilton path in $Y_{1}$ from $v_{1}$ to some $u_{1} \in A_{1}$. Then add the matching edge from $u_{1}$ into $B_{2}$. Keep doing this until we have a path from $u$ to $v$ that uses all the vertices of $Y_{0} \cup Y_{1} \cup \cdots \cup Y_{i}$.

If $i=t$, we have a Hamilton path connecting $u$ and $v$. If $i<t$, we extend the path as follows. Because $u, u_{0} \in A_{0}$ and $\left|A_{0}\right|=\left|B_{0}\right|$, there must be an edge of the form $w w^{\prime}$ in the path, where both $w, w^{\prime} \in B_{0}$. Remove this edge from the path and add on the matching edges from $w, w^{\prime}$ to $x, x^{\prime}$ in $A_{t}$. Then join them by a Hamilton path in $Y_{t}$ between $x$ and $x^{\prime}$. We can keep repeating this until we have a Hamilton path joining $u$ and $v$. This completes the proof.

The following two lemmas are based on key ideas used by Chen and Quimpo in their proof of Theorem 1.7 and is related to the first part of Lemma 2.5

Lemma 2.6. The cartesian product $P_{n} \square K_{2}$, where $P_{n}$ denotes the path of order $n$, has the property that if $u$ is any corner vertex - a vertex of valency 2-and $v$ is any vertex whose distance from $u$ is odd, then there is a Hamilton path in $P_{n} \square K_{2}$ joining $u$ and $v$.

Proof. The proof is trivial.

Lemma 2.7. The cartesian product $P_{n} \square C_{m}$ of a path of order $n, n \geqq 2$, and a cycle of order $m, m \geqq 3$, is Hamilton-laceable when $m$ is even and Hamilton-connected when $m$ is odd.

Proof. The graph $P_{2} \square C_{m}$ is a Cayley graph on an abelian group so that Theorem 1.7 establishes the result when $n=2$. Let $n>2$ and assume the result holds for $n-1$. Let $u$ and $v$ be arbitrary vertices in $P_{n} \square C_{m}$, where they are in different parts of the bipartition 
when $m$ is even. Refer to the cycles corresponding to a fixed vertex of $P_{n}$ as columns (we are thinking of drawing the graph in the usual way with cartesian coordinates).

If neither $u$ nor $v$ lie in the $n$-th column, there is a path $Q$ spanning the vertices of the first $n-1$ columns whose terminal vertices are $u$ and $v$ by induction. The path $Q$ must use an edge $u_{n-2, t} u_{n-2, t+1}$ from that column because $m \geqq 3$. Remove the edge $u_{n-2, t} u_{n-2, t+1}$ from $Q$, add the edges $u_{n-2, t} u_{n-1, t}$ and $u_{n-2, t+1} u_{n-1, t+1}$, and then connect the two vertices of valency 1 by passing through all the vertices of the $n$-th column. We now have a Hamilton path whose terminal vertices are $u$ and $v$.

If exactly one of the vertices lies in the $n$-th column, say $u$, then use induction to get a path from $v$, spanning the first $n-1$ columns, that terminates at the vertex in column $n-1$ that is adjacent to one of neighbors of $u$ in the $n$-th column. It is obvious how to extend the path to a Hamilton path with terminal vertices $u$ and $v$.

Similarly, if both $u$ and $v$ lie in the $n$-th column, use induction to get a path from $u$ to $v$ that spans the last $n-1$ columns. Extend it to a Hamilton path by removing an edge from the second column and picking up all the vertices of the first column.

Represent $P_{n} \square C_{m}$ with cartesian coordinates in the usual way, that is, the vertices are labelled $\left\{u_{i, j}: 0 \leqq i \leqq n-1,0 \leqq j \leqq m-1\right\}$, with edges joining $u_{i, j}$ to $u_{i, j-1}$ and $u_{i, j+1}$ for all $i, j$, and an edge from $u_{i, j}$ to $u_{i+1, j}$ for all $i \neq n-1$ and all $j$. Let $M(i, k, d)$ denote the $m$-matching formed by the edges $u_{i, j} u_{i+k, j+d}$, where $k>0, i+k<n, 0 \leqq d<m$ and $j+d$ is reduced modulo $m$. Note that $M(i, 1,0)$, as $i$ runs from 0 through $n-2$, are the matching edges currently joining the column cycles.

Lemma 2.8. Let $P_{n} \square C_{m}$ be the cartesian product of the path of order $n$ and cycle of order $m$, where $n>1, m$ is even, and $m \geqq 4$. If $X$ is the graph obtained by adding the edges of the m-matching $M(i, k, d)$, where both $k$ and $d$ are odd, to $P_{n} \square C_{m}$, then for every vertex $v \in X$ such that $v \neq u_{i, 0}$, there is a Hamilton path in $X$ whose terminal vertices are $u_{i, 0}$ and $v$.

Proof. The subgraph $Y$ induced on columns $i, i+1, \ldots, i+k$ is isomorphic to a Cayley graph on an abelian group as observed in [10]. The subgraph $Y$ is not bipartite so that it is Hamilton-connected by Theorem 1.7.

We'll refer to $v$ as the target vertex. If $v$ lies in $Y$, then there is a Hamilton path spanning $Y$ whose terminal vertices are $u_{i, 0}$ and $v$. The path must use an edge in column $i$ and an edge in column $i+k$. Remove the two edges and take the edges into columns $i-1$ and columns $i+k+1$, respectively. It is then easy to connect the end vertices of these edges with paths spanning columns $0,1, \ldots, i-1$ and columns $i+k+1, \ldots, n-1$. This gives us a Hamilton path from $u_{i, 0}$ to $v$ in the original graph.

It is a simple matter to find the appropriate Hamilton path if the target vertex is outside of $Y$ by exploiting the fact that $Y$ is Hamilton-connected.

Lemma 2.9. If $X$ is a connected Cayley graph on the generalized dihedral group $D_{H}$ so that every element of the connection set lies in $H \tau$, then $X$ has a Hamilton path.

Proof. Let $S$ denote the connection set. If $|S|=1$, then $X$ must be $K_{2}$ which has a Hamilton path. If $|S|=2$, then $X$ itself must be a Hamilton cycle which certainly contains a Hamilton path. We proceed by induction and let $|S| \geqq 3$. 
Remove an arbitrary element $s$ from $S$. If the subgraph Cay $\left(D_{H}: S-s\right)$ is connected, then it has a Hamilton path by induction. If $\operatorname{Cay}\left(D_{H}: S-s\right)$ is not connected, then each component contains a Hamilton path by induction. The element $s$ cyclically joins the components $Y_{1}, Y_{2}, \ldots, Y_{t}$, where we may assume that $s$ joins vertices of $Y_{i} \cap H$ to vertices of $Y_{i+1} \cap H \tau$. Consider a Hamilton path in $Y_{t}$ with one terminal vertex $v_{t} \in H \tau$. Let $u_{t-1} \in Y_{t-1}$ be adjacent to $v_{t}$ via an $s$-edge. Because the components themselves are vertex-transitive, there is a Hamilton path in $Y_{t-1}$ with $u_{t-1}$ as a terminal vertex. We then join these two paths via the $s$-edge and get a path spanning $Y_{t-1} \cup X_{t}$. We continue this way until we obtain a Hamilton path.

\section{Honeycomb toroidal graphs}

In this section we study Hamilton paths in honeycomb toroidal graphs.

Definition 3.1. A Hamilton path from $u_{0,0}$ to $u_{0, j}$ in $\operatorname{HTG}(m, 2 n, s)$ is called twisted if the edge incident with $u_{0,0}$ is a jump edge and the edge incident with $u_{0, j}$ is a flat edge.

The next lemma gives us a tool for going from solutions for particular honeycomb toroidal graphs to solutions for a much wider class of honeycomb toroidal graphs. Some observations about Hamilton paths in honeycomb toroidal graphs are in order. If we have a Hamilton path $P$ from $u_{0,0}$ to some vertex $v$ and there is a flat edge from $u_{i-1, j}$ to $u_{i, j}$ in $P$, then there must be a flat edge or jump edge incident with $u_{i, j+1}$ or $u_{i, j-1}$ unless one of these vertices is the other terminal vertex of $P$ and it lies in the last column. The point is that flat edges in a Hamilton path force other flat edges or jump edges also to be in the path with the exception of very special situations.

Lemma 3.2. If there is a Hamilton path from $u_{0,0}$ to $u_{1, j}$ in $\operatorname{HTG}(2,2 n, s)$ that uses both flat edges and jump edges, then there is a Hamilton path from $u_{0,0}$ to $u_{i, j}$ in $\operatorname{HTG}(m, 2 n, s)$ for all odd $i$ satisfying $0<i<m$ and all even $m \geqq 2$. Similarly, if there is a non-twisted Hamilton path from $u_{0,0}$ to $u_{0, j}$ in $\operatorname{HTG}(2,2 n, s)$ that uses both flat edges and jump edges, then there is a Hamilton path from $u_{0,0}$ to $u_{i, j}$ in $\operatorname{HTG}(m, 2 n, s)$ for all even $i$ satisfying $0 \leqq i<m$ and all even $m \geqq 2$.

Proof. If $s=0$ and $m=2$, the jump is zero and flat edges and jump edges look the same. However, in this case we still distinguish between them. Edges joining $u_{0, j} u_{1, j}$ are flat when $j$ is odd and jump edges when $j$ is even. With this in mind, all that follows works for this special case too.

The idea behind the proof is to show that we can take a Hamilton path in $\operatorname{HTG}(m, 2 n, s)$ and obtain a Hamilton path in $\operatorname{HTG}(m+2,2 n, s)$ with appropriate end vertices. Suppose we have a Hamilton path $P$ from $u_{0,0}$ to $u_{i, j}$ in $\operatorname{HTG}(m, 2 n, s), i>0$, and that $u_{0, k_{1}} u_{1, k_{1}}, u_{0, k_{2}} u_{1, k_{2}}, \ldots, u_{0, k_{t}} u_{1, k_{t}}, 0<k_{1}<k_{2}<\cdots<k_{t} \leqq 2 n-1$, are the flat edges of $P$ between the columns indexed by 0 and 1 , respectively (there must be at least one flat edge). We insert two new columns between the columns indexed by 0 and 1 which we temporarily index with $\alpha$ and $\beta$ from left to right. Replace the flat edge $u_{0, k_{1}} u_{1, k_{1}}$ with the path

$$
u_{0, k_{1}} u_{\alpha, k_{1}} u_{\alpha, k_{1}+1} \cdots u_{\alpha, k_{2}-1} u_{\beta, k_{2}-1} u_{\beta, k_{2}-2} \cdots u_{\beta, k_{1}} u_{1, k_{1}} .
$$

We then do the same for each flat edge, that is, we work from the row index of the flat edge up to one less than the row index of the next (in cyclic order modulo $2 n$ ) flat edge, and obtain a Hamilton path with one terminal vertex being $u_{0,0}$ and two new columns inserted 


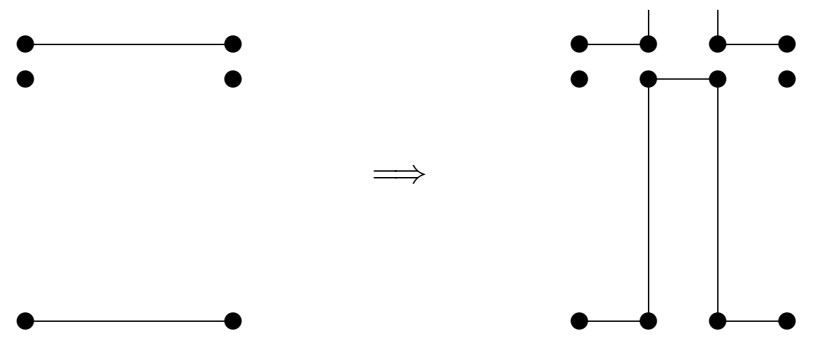

Figure 1: Inserting two new columns.

to the left of the other terminal vertex. Upon reindexing the columns, we have a Hamilton path from $u_{0,0}$ to $u_{i+2, j}$ in $\operatorname{HTG}(m+2,2 n, s)$. (See Figure 1 for a schematic of how the procedure works.)

If we have a Hamilton path $P$ from $u_{0,0}$ to $u_{i, j}$ in $\operatorname{HTG}(m, 2 n, s)$ and $i<m-1$, then we can insert two columns to the right of the column indexed $i$ by using the flat edges of $P$ between the columns indexed $m-2$ and $m-1$. Hence, there is a Hamilton path from $u_{0,0}$ to $u_{i, j}$ in $\operatorname{HTG}(m+2,2 n, s)$.

There are two special cases we must consider. Suppose there is a Hamilton path $P$ from $u_{0,0}$ to $u_{m-1, j}$ in $\operatorname{HTG}(m, 2 n, s)$ and we want a Hamilton path in $\operatorname{HTG}(m+2,2 n, s)$ from $u_{0,0}$ to $u_{m-1, j}$. Thus, we want to insert two columns to the right of the column indexed $m-1$ in $\operatorname{HTG}(m, 2 n, s)$ while keeping the terminal vertex of a new Hamilton path in the same column. We use jump edges to do this. Let $u_{m-1, k_{1}}, u_{m-1, k_{2}}, \ldots, u_{m-1, k_{t}}$, $0 \leqq k_{1}<k_{2}<\cdots<k_{t}<2 n-1$, be the vertices in the column indexed $m-1$ that are incident with jump edges of $P$. We insert two new columns on the right that will be indexed $m$ and $m+1$. Detach the jump edge that is incident with $u_{m-1, k_{1}}$ and make it incident with $u_{m+1, k_{1}}$. Now take the path

$$
u_{m-1, k_{1}} u_{m, k_{1}} u_{m, k_{1}+1} \cdots u_{m, k_{2}-1} u_{m+1, k_{2}-1} u_{m+1, k_{2}-2} \cdots u_{m+1, k_{1}}
$$

from $u_{m-1, k_{1}}$ to $u_{m+1, k_{1}}$ through the two new columns. If we do this for each jump edge, we get a Hamilton path from $u_{0,0}$ to $u_{m-1, j}$ in $\operatorname{HTG}(m+2,2 n, s)$.

For the final special case, suppose we have a Hamilton path $P$ from $u_{0,0}$ to $u_{0, j}$ in $\operatorname{HTG}(m, 2 n, s)$ and we want a Hamilton path from $u_{0,0}$ to $u_{2, j}$ in $\operatorname{HTG}(m+2,2 n, s)$. If the terminal edge of $P$ is a vertical edge, we add the flat edge $u_{0, j} u_{1, j}$ to $P$ and now use the flat edges of $P$, together with the new flat edge, to insert two new columns between the columns indexed 0 and 1 . We make one small adjustment to the insertion procedure, namely, we don't include the new flat edge between $u_{1, j}$ and the vertex in the new column to the left. Thus, upon reindexing the columns, we have a Hamilton path from $u_{0,0}$ to $u_{2, j}$ in $\operatorname{HTG}(m+2,2 n, s)$.

If the terminal edge of $P$ is $u_{1, j} u_{0, j}$, the extension is more complicated. Because we may assume that $P$ is non-twisted, we know that the initial edge of $P$ is either $u_{0,0} u_{0,1}$ or $u_{0,0} u_{0, n-1}$. We insert two columns to the left of the column indexed 0 and index them $\alpha$ and $\beta$ from left to right. Let $u_{0, k_{1}}, u_{0, k_{2}}, \ldots, u_{0, k_{t}}, 0<k_{1}<k_{2} \cdots k_{2}<n-1$, be the vertices in the column indexed 0 that are incident with jump edges in $P$. Insert the path

$$
u_{\alpha, 0} u_{\alpha, 1} \cdots u_{\alpha, k_{1}-1} u_{\beta, k_{1}-1} u_{\beta, k_{1}-2} \cdots u_{\beta, 0} u_{0,0}
$$


into the new graph. For each of the other vertices $u_{0, k_{r}}$, detach the jump edge incident with $u_{0, k_{r}}$ and attach it to $u_{\alpha, k_{r}}$, and insert a path from $u_{\alpha, k_{r}}$ to $u_{0, k_{r}}$ similar to the path from $u_{\alpha, 0}$ to $u_{0,0}$. After reindexing the columns we have a Hamilton path from $u_{0,0}$ to $u_{2, j}$ in $\operatorname{HTG}(m+2,2 n, s)$.

The above operations allow us to insert an even number of columns to the left and to the right of a column containing the terminal vertex of a Hamilton path starting at $u_{0,0}$.

Note that the insertion procedure used in the preceding proof, never introduces twisted Hamilton paths, that is, if a Hamilton path is not twisted, then after making any of the insertions the resulting Hamilton path is not twisted.

We now want to prove a theorem about honeycomb toroidal graphs that will be used as a building block for the proof of the main result. However, before doing so we prove a lemma that will simplify the exposition.

Definition 3.3. A path $P$ in $\operatorname{HTG}(2,2 n, s)$ with terminal vertices $u$ and $v$ is said to be vertically extendable when it satisfies the following two properties:

1. $u_{0, j}$ belongs to $P$ if and only if $u_{1, j}$ belongs to $P$, and

2. for each maximal segment $u_{0, i_{1}}, u_{0, i_{1}+1}, \ldots, u_{0, i_{1}+k}$ of vertices not belonging to $P$, exactly one vertex from $\left\{u_{0, i_{1}-1}, u_{0, i_{1}+k+1}\right\}$ is incident with a flat edge of $P$ and the other vertex is incident with a jump edge, although the jump edge need not belong to $P$.

Note that the definition implies that a given flat edge cannot arise as the flat edge for two different maximal segments not belonging to $P$. This property is used in the proof of the next lemma.

Lemma 3.4. If $P$ is a vertically extendable path in $\operatorname{HTG}(2,2 n, s)$ with terminal vertices $u$ and $v$, then there is a Hamilton path in $\operatorname{HTG}(2,2 n, s)$ with terminal vertices $u$ and $v$.

Proof. Let $P$ be the path described in the hypotheses. If $P$ already is a Hamilton path, then there is nothing to prove. Otherwise, consider a maximal segment $u_{0, i_{1}}, u_{0, i_{1}+1}, \ldots$, $u_{0, i_{1}+k}$ of vertices not belonging to $P$. Without loss of generality assume that

$$
u_{0, i_{1}+k+1} u_{1, i_{1}+k+1}
$$

is the flat edge belonging to $P$. Replace this flat edge (see Figure 2) with the path

$$
u_{0, i_{1}+k+1} u_{0, i_{1}+k} u_{0, i_{1}+k-1} \ldots u_{0, i_{1}} u_{1, i_{1}} u_{1, i_{1}+1} \ldots u_{1, i_{1}+k+1} .
$$

If the unique flat edge had been $u_{0, i_{1}-1} u_{1, i_{1}-1}$, we make the obvious modification.

We perform the above operation for each unique flat edge associated with the maximal segments of vertices not belonging to $P$. We have a Hamilton path and the terminal vertices have not been altered. This completes the proof.

In the subsequent material, when we apply Lemma 3.4 to go from a path $P$ with terminal vertices $u$ and $v$ to a Hamilton path with terminal vertices $u$ and $v$, we shall say that we have extended $P$ to a Hamilton path by vertical extension. Note that any jump edges appearing in $P$ remain in the vertical extension to a Hamilton path. 


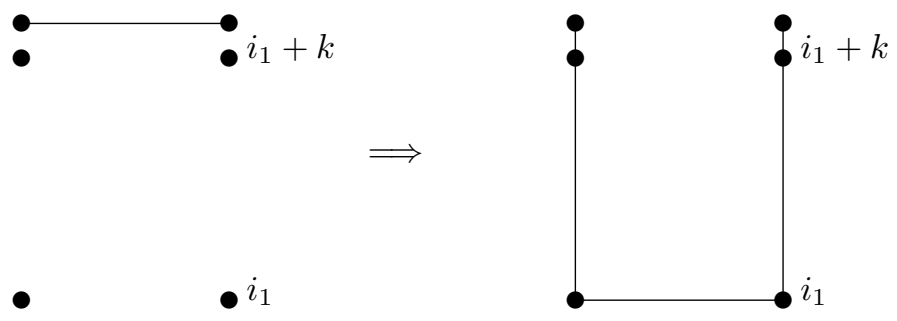

Figure 2: Typical vertical extension insertion.

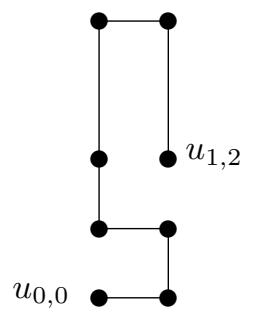

Figure 3: Typical Hamilton path when $s=0$.

Theorem 3.5. The honeycomb toroidal graph $\operatorname{HTG}(m, 2 n, s)$ is Hamilton-laceable whenever $m$ is even.

Proof. Since the graph $\operatorname{HTG}(m, 2 n, s)$ is bipartite and vertex-transitive, it suffices to find a Hamilton path from $u_{0,0}$ to any vertex $u_{i, j}$ with $i+j$ odd. Because of Lemma 3.2, it then suffices to prove the following two facts: (1) there is a Hamilton path from $u_{0,0}$ to $u_{1, j}, j$ even, in $\operatorname{GHT}(2,2 n, s)$ that has both flat edges and jump edges; and (2) there is a non-twisted Hamilton path from $u_{0,0}$ to $u_{0, j}, j$ odd, in $\operatorname{GHT}(2,2 n, s)$ that uses both flat and jump edges.

We handle the case that $s=0$ first. The path starting $u_{0,0} u_{0,2 n-1} u_{1,2 n-1}$ and now works down going back and forth between the two columns terminates at $u_{1,0}$. For all other vertices $u_{1, j}, j>0$ even, we start the path $u_{0,0} u_{1,0}$ and then work up going back and forth between the two columns until we reach vertex $u_{0, j-1}$. We then go straight up the column indexed 0 to $u_{0,2 n-1}$, across to the other column and down to $u_{1, j}$ (see Figure 3 ). For vertices $u_{0, j}, j$ odd and $j \neq 2 n-1$, in the column indexed 0 , start working up going back and forth between the two columns until reaching $u_{1, j-1}$. Then go to the top, across to $u_{0,2 n-1}$, and then down to $u_{0, j}$. The latter construction does not work for $u_{0,2 n-1}$ because the resulting Hamilton path is twisted.

For the special case $u_{0,2 n-1}$, start with $u_{0,0} u_{1,0} u_{1,2 n-1}$. Then continue down the column indexed 1 until reaching $u_{1,1}$. Then take the edge to $u_{0,1}$ followed by going up the column indexed 0 until reaching $u_{0,2 n-1}$. The resulting Hamilton path from $u_{0,0}$ to $u_{0,2 n-1}$ uses one flat edge, one jump edge, and is not twisted. We have obtained Hamilton paths from $u_{0,0}$ to all target vertices that use both flat and jump edges. None of the Hamilton paths are twisted and this completes the case for $s=0$.

Henceforth, assume $s>0$. For $i=0,1, \ldots, n-1$, let $Q(i)$ denote the 
3-path

$$
u_{0,2 i} u_{0,2 i+1} u_{1,2 i+1} u_{1,2 i}
$$

and $R(i)$ denote the 3-path

$$
u_{0,2 i} u_{0,2 i-1} u_{1,2 i-1} u_{1,2 i} .
$$

The subgraph formed by the jump edges and the edges of $Q(0), Q(1), \ldots$, $Q(n-1)$ is spanning and regular of valency 2. Furthermore, a vertical cyclic shift by two is an automorphism of the subgraph. Thus, its components are cycles of the same length and the number of them is $d=\operatorname{gcd}(n, s / 2)$. Note that the jump edge $u_{1,2 i} u_{0,2 i+s}$ joins the terminal vertex of $Q(i)$ to the initial vertex of $Q(i+(s / 2))$. Let $C(i)$ denote the cycle containing $Q(i)$ for $i=0,1, \ldots, d-1$. The subgraph formed by the jump edges and the edges of $R(0), R(1), \ldots, R(n-1)$ behaves in the same way with $d$ cycles for its components as well. Let $C^{\prime}(i)$ denote the cycle containing $R(i)$ for $i=0,1, \ldots, d-1$.

Let $u_{1, j}$ be any vertex, with $j$ even, from the column indexed by 1 . Suppose $u_{1, j}$ lies on $C(k)$. Starting at $u_{0,0}$, traverse the entire cycle $C(0)$ and terminate at $u_{0,1}$. Then add the edge $u_{0,1} u_{0,2}$. Now traverse the cycle $C(1)$ from $u_{0,2}$ to $u_{0,3}$. Then add the edge to $u_{0,4}$ in $C(2)$. Continue in this way obtaining a path from $u_{0,0}$ to $u_{0,2 k}$ that uses all the vertices of $C(0) \cup C(1) \cdots \cup C(k-1)$. Now from $u_{0,2 k}$ traverse $C(k)$, starting with edge $u_{0,2 k} u_{0,2 k+1}$, until reaching $u_{1, j}$. The path we have constructed from $u_{0,0}$ to $u_{1, j}$ is easily seen to be vertically extendable. Thus, we obtain a Hamilton path $P\left(u_{1, j}\right)$ from $u_{0,0}$ to $u_{1, j}$ by vertical extension.

With the exception of $P\left(u_{1,0}\right)$, each of the Hamilton paths just constructed has both flat edges and jump edges. The Hamilton path $P\left(u_{1,0}\right)$ has no jump edges because it is the vertical extension of $Q(0)$. The vertex $u_{1,0}$ must be treated individually.

If $d=1$, that is, $C(0)$ itself is a Hamilton cycle, then $C^{\prime}(0)$ also is a Hamilton cycle. Build a path as follows. Start the path with the four vertices $u_{0,0} u_{0,2 n-1} u_{1,2 n-1} u_{1,2 n-2}$. Now traverse the cycle $C^{\prime}(0)$ starting with the edge from $u_{1,2 n-2}$ to $u_{1,2 n-3}$. Because $C^{\prime}(0)$ is a Hamilton cycle, as we traverse it in the indicated direction, when we reach the vertex $u_{1,0}$ along the edge from $u_{0, s}$, all vertices are distinct so that we have a path from $u_{0,0}$ to $u_{1,0}$ that uses both flat edges and jump edges. It is vertically extendable so that the subcase of $d=1$ is finished.

Build a path as follows when $d>1$. Start the path with the vertices $u_{0,0} u_{0,2 n-1} u_{1,2 n-1}$ $u_{1,2 n-2}$. From the vertex $u_{1,2 n-2}$ traverse the entire cycle $C^{\prime}(d-1)$ to the vertex $u_{1,2 n-3}$. From the vertex $u_{1,2 n-3}$ take the edge to $u_{1,2 n-4}$. From this vertex we traverse the entire cycle $C^{\prime}(d-2)$ to the vertex $u_{1,2 n-5}$. We continue in this way until the cycles $C^{\prime}(d-1)$, $C^{\prime}(d-2), \ldots, C^{\prime}(1)$ have been traversed and we are at the vertex $u_{1,2 n-2 d}$ which lies on $C^{\prime}(0)$. We now traverse $C^{\prime}(0)$ in the direction that allows us to reach $u_{1,0}$ along the edge $u_{1,0} u_{0, s}$. This path has both jump edges and flat edges. Vertical extension then gives us a Hamilton path from $u_{0,0}$ to $u_{1,0}$ with flat edges and jump edges.

We now have Hamilton paths from $u_{0,0}$ to every vertex of the form $u_{1, j}, j$ even, with flat edges and jump edges. We now turn our attention to vertices of the form $u_{0, j}, j$ odd.

When $d=1$, a Posa exchange for $P\left(u_{1, k}\right)$ on the edge $u_{1, k} u_{0, k+s}$ produces a Hamilton path from $u_{0,0}$ to $u_{0, k+s+1}$. All of these Hamilton paths have flat edges and jump edges. None of them are twisted except the Hamilton path that terminates at $u_{0,1}$. Thus, we need to find a non-twisted Hamilton path from $u_{0,0}$ to $u_{0,1}$.

For this special case we use the 3-paths $R(0), R(1), \ldots, R(n-1)$ to form the Hamilton path from $u_{0,0}$ to $u_{1,2-s}$ noting that $u_{0,1}$ is the successor of $u_{0,2}$. When we perform the 
Posa exchange on the edge $u_{1,2-s} u_{0,2}$, we obtain a non-twisted Hamilton path from $u_{0,0}$ to $u_{0,1}$.

We now have found Hamilton paths of the desired type from $u_{0,0}$ to all vertices of the form $u_{0, j}, j$ odd, when $d=1$. We proceed slightly differently for $d>1$.

Assume $d>1$. A Posa exchange for $P\left(u_{1, j}\right)$ on the edge $u_{1, j} u_{0, j+s}$ produces a nontwisted Hamilton path from $u_{0,0}$ to $u_{0, j+s-1}$ whenever $u_{0, j+s}$ is a vertex of $C(k)$, where $k \neq 0$ and $u_{0, j+s} \neq u_{0,2 k}$. For the special cases of $u_{0,2 k-1}, 0<k \leqq d-1$, build the required Hamilton path as follows. Traverse all of $C(0)$ from $u_{0,0}$ to $u_{0,1}$. If $k=1$, then $u_{0,2 k-1}=u_{0,1}$ and the latter path is vertically extendable to a non-twisted Hamilton path from $u_{0,0}$ to $u_{0,1}$. If $k>1$, continue building the path by adding the edge $u_{0,1} u_{0,2}$, and then traversing the entire cycle $C(1)$ until reaching the vertex $u_{0,3}$. If $k=2$, then $u_{0,2 k-1}=u_{0,3}$ and we use vertical extension to get a non-twisted Hamilton path from $u_{0,0}$ to $u_{0,3}$. It is obvious how to continue this so that we obtain non-twisted Hamilton paths from $u_{0,0}$ to vertices of the form $u_{0,2 k-1}, 0<k \leqq d-1$.

We are left with the requirement of finding appropriate Hamilton paths from $u_{0,0}$ to vertices of the form $u_{0, j}$, where $j$ is odd and $u_{0, j+1}$ belongs to $C(0)$. Traverse all of $C^{\prime}(0)$ from $u_{0,0}$ to $u_{0,2 n-1}$. The vertical extension of this path is a non-twisted Hamilton path from $u_{0,0}$ to $u_{0,2 n-1}$ containing both flat edges and jump edges.

Let $u_{0, j}$ be any vertex of the form $u_{0, j}$, where $j$ is odd and $u_{0, j+1}$ belongs to $C(0)$, distinct from $u_{0,2 n-1}$. Traverse $C^{\prime}(0)$ starting at $u_{0,0}$ along the edge $u_{0,0} u_{0,2 n-1}$ until reaching the vertex $u_{1, j+1-s}$. The resulting path is vertically extendable to a Hamilton path joining $u_{0,0}$ and $u_{1, j+1-s}$. Now perform a Posa exchange on the resulting Hamilton path using the edge $u_{1, j+1-s} u_{0, j+1}$. The result is a non-twisted Hamilton path from $u_{0,0}$ to $u_{0, j}$ that has both flat edges and jump edges. This completes the proof.

\section{Proof of main theorem}

The proof of the main theorem is divided into three cases: $S_{1}=\emptyset,\left|S_{1}\right|=1$, and $\left|S_{1}\right| \geqq$ 2 . The preceding section contains a proof that honeycomb toroidal graphs with an even number of columns are Hamilton-laceable. This forms the basis for the proof of Theorem 1.8 when $S_{1}=\emptyset$. The other two cases rely on the lemmas proved in Section 2. As a reminder, let $X=\operatorname{Cay}\left(D_{H} ; S\right)$ be a Cayley graph on a generalized dihedral group $D_{H}$ such that $D_{H}=\langle H, \tau\rangle,\left|D_{H}\right| \equiv 0(\bmod 4)$, Cay $\left(D_{H} ; S\right)$ is connected, and $|S| \geqq 3$. Recall that $S_{1}=S \cap H$ and $S_{2}=S \cap H \tau$.

Case 1. Let $S_{1}=\emptyset$.

The most we can hope for is that $X$ is Hamilton-laceable because it is bipartite. We prove this case by induction on $\left|S_{2}\right|$ with the base for the induction being $\left|S_{2}\right|=3$, where all three elements belong to $H \tau$. Every element of $S$ is an involution and produces a 1factor in $X$. Let $I_{j}$ denote the 1 -factor in $X$ generated by $\tau_{j}, j=1,2,3$. Let $F_{i j}$ denote the 2-factor in $X$ arising from $I_{i} \cup I_{j}$. Note that $F_{i j}$ is composed of vertex-disjoint cycles each of which has length $2\left|\tau_{i} \tau_{j}\right|$.

Let $C(1)$ and $C(2)$ be two of the cycles comprising $F_{i j}$. If a vertex of $C(1)$ is adjacent to a vertex of $C(2)$ via an edge of $I_{k}$, where $I_{k}$ is the other 1-factor, then because $\tau_{i} \tau_{j} \tau_{k}=$ $\tau_{k} \tau_{j} \tau_{i}$, it is easy to see that alternate vertices of $C(1)$ are adjacent to alternate vertices of $C(2)$. Because $X$ is connected, it is easy to see that $X$ is a honeycomb toroidal graph $\operatorname{HTG}(m, 2 n, s)$ with the cycles of $F_{i j}$ being the vertical columns and the edges of $I_{k}$ being the flat edges and jump edges. Note that $n=\left|\tau_{i} \tau_{j}\right|$ and $m$ is the number of components of 
$F_{i j}$.

Thus, if $F_{12}$ has an even number of components, $X$ is Hamilton-laceable by Theorem 3.5. So we suppose that $F_{12}$ is $\operatorname{HTG}(m, 2 n, s)$, where $m$ is odd and $n=\left|\tau_{1} \tau_{2}\right|$. Because $\left|D_{H}\right|$ is a multiple of 4 , we then must have that $n$ is even.

Now consider the components of $F_{13}$. If we start at a vertex of the first column in $\operatorname{HTG}(m, 2 n, s)$ and alternately travel by $\tau_{1}$ and $\tau_{3}$, when we return to the first column we have travelled by $s+m$ around the cycle in a cyclic fashion. In other words, we have jumped by $(s+m) / 2$ between edges of $I_{1}$. Hence, the number of components of $F_{13}$ is $\operatorname{gcd}(n,(s+m) / 2)$. Similarly, the number of components of $F_{23}$ is $\operatorname{gcd}(n,(s-m) / 2)$. Because $m$ is odd, $(s+m) / 2$ and $(s-m) / 2$ have opposite parity. Then $n$ even implies that one of $F_{13}$ or $F_{23}$ has an even number of components. The resulting honeycomb toroidal graph has an even number of columns and Theorem 3.5 implies that $X$ is Hamiltonlaceable.

Therefore, we conclude that $X$ is Hamilton-laceable when $S$ consists of three involutions belonging to $H \tau$. Hence, we assume $|S|>3$.

If there is some $s \in S$ such that $\operatorname{Cay}\left(D_{H} ; S-s\right)$ is connected, then this subgraph is Hamilton-laceable by induction. Hence, we may assume that $S-s$ generates a proper subgroup of $D_{H}$ for all $s \in S$. Consider $X^{\prime}=\operatorname{Cay}\left(D_{H} ; S-s\right)$ for a particular $s \in S$. The Cayley graph $X^{\prime}$ is disconnected with components all isomorphic to $Y=\operatorname{Cay}(\langle S-$ $s\rangle ; S-s)$. If $Y$ has order divisible by 4 , then $Y$ is Hamilton-laceable by induction. Let $Y_{1}$, $Y_{2}, \ldots, Y_{m}$ be the components of $X$ with respective bipartitions $\left(A_{1}, B_{1}\right),\left(A_{2}, B_{2}\right), \ldots$, $\left(A_{m}, B_{m}\right)$. Each part $A_{i}$ and $B_{i}, i=1,2, \ldots, m$, is a coset of a subgroup of $H$. Hence, by Lemma 2.2, we may assume that the subscripts have been chosen so that there is a perfect matching joining vertices of $A_{i}$ and vertices of $B_{i+1}$, for $i=1,2, \ldots, m-1$, and vertices of $A_{m}$ with vertices of $B_{1}$. We then may apply Lemma 2.3 to conclude that $X$ is Hamilton-laceable.

From the preceding, we see that it suffices to show that for some $s \in S$ the order of the components of $\operatorname{Cay}\left(D_{H} ; S-s\right)$ is a multiple of 4. Choose an arbitrary $s_{1} \in S$. The components of $\operatorname{Cay}\left(D_{H} ; S-s_{1}\right)$ must have even order because each element of $S$ generates a 1-factor. If $\operatorname{Cay}\left(D_{H} ; S-s_{1}\right)$ has components whose order is not a multiple of 4 , then there must be an even number of components. The edges generated by $s_{1}$ join these components in a cyclic fashion so that if we now consider $S-s_{2}$, where $s_{2} \neq s_{1}$, then each component of $\operatorname{Cay}\left(D_{H} ; S-s_{2}\right)$ intersects each component of $\operatorname{Cay}\left(D_{H} ; S-s_{1}\right)$ in the same even number of vertices so that $\operatorname{Cay}\left(D_{H} ; S-s_{2}\right)$ has components whose order is divisible by 4 . This completes the proof of Case 1 .

Case 2. Let $\left|S_{1}\right|=1$.

Let $X_{1}$ be the subgraph induced by $X$ on $H$. If $X_{1}$ is connected, then $X$ must be $K_{4}$ which certainly is Hamilton-connected. Hence, we assume that $X_{1}$ is not connected so that $h$ generates a non-trivial 1 -factor in both $X_{1}$ and $X_{2}$. Note that $h$ commutes with every element in $S_{2}$ because $h\left(h_{i} \tau\right)=h_{i} h \tau=h_{i} \tau h^{-1}=\left(h_{i} \tau\right) h$. This is a useful fact.

Another useful fact is the following, namely, we prove that $X$ is bipartite if and only if removing $h$ from $S$ disconnects the graph, that is, $X^{\prime}=\operatorname{Cay}\left(D_{H} ; S-h\right)$ is not connected. Assume that $X^{\prime}$ is connected and let $P$ be a path joining $u$ to $u h$ in $X^{\prime}$. The path $P$ must have even length because $X^{\prime}$ is bipartite and $u$ and $u h$ lie in the same part. Joining $u$ to $u h$ gives an odd length cycle in $X$ so that $X$ is not bipartite. On the other hand, if $X^{\prime}$ is not connected, then for each edge $u v$ in a fixed component $C$ of $X^{\prime}$, there is an edge joining $u h$ to $v h$ in $X^{\prime}$. This implies that all of the edges from $C$ generated by $h$ in $X$ go to another 
single component of $X^{\prime}$. Hence, $X^{\prime}$ has exactly two components each of which is bipartite. It is easy to see that $X$ too is bipartite.

Let $\bar{X}$ denote the quotient graph obtained by contracting each edge of the perfect matching generated by $h$ to a vertex. The graph $\bar{X}$ is a Cayley graph on a generalized dihedral group. It has a Hamilton path by Lemma 2.9. Hence, in $X$ there is a spanning subgraph isomorphic to $P_{n} \square K_{2}$. Lemma 2.6 implies that there is a Hamilton path from a corner vertex to any vertex at odd distance from it. Thus, if $X$ is bipartite, there is a Hamilton path from a corner vertex to every vertex in the other part of the bipartition. Because $X$ is vertex-transitive, this is true for all vertices and we conclude that $X$ is Hamilton-laceable.

Because of the preceding paragraph, we may assume that $X$ is not bipartite. If the quotient graph $\bar{X}$ has order a multiple of 4 , then it has a Hamilton cycle by Case 1 . Thus, the original graph $X$ has a spanning subgraph satisfying the hypotheses of Lemma 2.4. This lemma then implies that $X$ is Hamilton-connected.

This leaves us with the subcase that $X$ is not bipartite and the order of $\bar{X}$ is of the form $2 t$, where $t$ is odd. This subcase takes more work.

We induct on the cardinality of $S_{2}$. When $\left|S_{2}\right|=2$, the quotient graph $\bar{X}$ must be a Hamilton cycle because $X$ is connected. This implies that $X$ has a spanning subgraph satisfying the hypotheses of Lemma 2.4 which, in turn, implies that $X$ is Hamilton-connected.

Assume that $\left|S_{2}\right|=r>2$ and the result holds whenever $2 \leqq\left|S_{2}\right|<r$. Choose $s \in S_{2}$ and consider the subgraph $X^{\prime}=\operatorname{Cay}\left(D_{H} ; S-s\right)$. If $X^{\prime}$ is connected and not bipartite, then it is Hamilton-connected by induction.

Next assume that $X^{\prime}$ is connected but bipartite. From the argument above, we know that deleting the edges generated by $h$ disconnects $X^{\prime}$. Because $h$ commutes with all the elements of $S_{2}$, the removal of the edges generated by $h$ produces two components $\mathbb{C}_{1}$ and $\mathbb{C}_{2}$. By Lemma 2.9, the subgraph $\mathbb{C}_{1}$ has a Hamilton path. Using the Hamilton path and the edges generated by $h$, we get a spanning subgraph $Y^{\prime}$ of $X^{\prime}$ isomorphic to $P_{2 t} \square K_{2}$.

Label the vertices of $Y^{\prime}$ with $u_{i, j}$, where $0 \leqq i<2 t, j=0,1, u_{i, 0} u_{i, 1}$ are the edges generated by $h$, and $u_{i, j}$ is adjacent to $u_{i+1, j}$ for $i=0,1, \ldots, 2 t-2$. Lemma 2.6 tells us there is a Hamilton path from $u_{0,1}$ to any vertex whose distance from $u_{0,1}$ in $Y^{\prime}$ is odd.

Because the graph $X$ is not bipartite, the deletion of the edges generated by $h$ does not disconnect $X$. This implies that the edges generated by $s$ join vertices of $\mathbb{C}_{1}$ to vertices of $\mathbb{C}_{2}$. Therefore, if we take the subgraph $Y^{\prime}$ and add the edges generated by $s$ from the vertices $u_{0,0}$ and $u_{0,1}$, we have a 2-matching of the form $M(0, k, 1), k$ odd (in the notation of Lemma 2.8). Let $Y^{*}$ be the graph obtained by augmenting $Y^{\prime}$ with the 2-matching $M(0, k, 1)$. The subgraph of $Y^{*}$ from $i=0$ up through $i=k$ is Hamilton-connected by Lemma 2.4. Using this fact and Lemma 2.6 it is easy to show there is a Hamilton path from $u_{0,1}$ to any vertex $v$. other than $v=u_{k, 0}$. Thus, for every vertex $v \in X, v \neq u_{0,1}$, there is a Hamilton path with terminal vertices $u_{0,1}$ and $v$. Because $X$ is vertex-transitive, $X$ is Hamilton-connected.

We now move to the subcase that $X^{\prime}$ is not connected. If the components of $X^{\prime}$ are Hamilton-connected, then Lemma 2.5 implies that $X$ is Hamilton-connected. Hence, we assume the components of $X^{\prime}$ are bipartite. This subcase is similar to the immediately preceding one. Because the components $\mathbb{C}_{0}, \mathbb{C}_{1}, \ldots, \mathbb{C}_{r}$ are bipartite, each may be written as $\mathbb{C}_{i}=\left(A_{i}, B_{i}, C_{i}, D_{i}\right)$, where $h$ edges join $A_{i}$ to $B_{i}$ and $C_{i}$ to $D_{i}$, and elements of $S_{2}$ generate edges joining $A_{i}$ to $C_{i}$ and $B_{i}$ to $D_{i}$. We may then assume the subscripts are chosen so that edges generated by $s$ join vertices of $A_{i}$ to vertices of $C_{i+1}$, and vertices of 
$B_{i}$ to vertices of $D_{i+1}$ for $i=0,1, \ldots, r-1$. Because $X$ is not bipartite, we must have $s$ producing edges joining $A_{r}$ to $D_{0}$ and $B_{r}$ to $C_{0}$.

We use Lemma 2.9 to get a path spanning $A_{i} \cup C_{i}$ for $i=0,1, \ldots, r$. Because $A_{i} \cup C_{i}$ is vertex-transitive, we may choose the beginning of the Hamilton path in $A_{i} \cup C_{i}$ to be the vertex incident with the edge generated by $s$ that is incident with the terminal vertex of the Hamilton path in $A_{i+1} \cup C_{i+1}$. In this way we use edges generated by $s$ to produce a path spanning all the vertices of

$$
A_{0} \cup A_{1} \cup \cdot \cup A_{r} \cup C_{0} \cup C_{1} \cup \cdots \cup C_{r} .
$$

We now use the edges generated by $h$ to obtain a spanning subgraph $Y$ of $X$ isomorphic to $P_{2 t} \square K_{2}$.

The subgraph $Y$ is bipartite, but each time we use an edge generated by $s$, the parts in the bipartition of a component are interchanged. Because each component has an even number of $h$-edges and the total number of $h$-edges is $2 t$ with $t$ odd. There must be an odd number of components. Hence, if we add the $s$-edges from the first edge of $Y$ (from component $\mathbb{C}_{r}$ ) to some $h$-edge from component $\mathbb{C}_{0}$ we violate the bipartition, that is, we have a matching $M(0, k, 1)$ for some odd $k$. We then obtain that $X$ is Hamilton-connected as in the preceding subcase. This completes Case 2.

Case 3. Let $\left|S_{1}\right| \geqq 2$.

When $X_{1}$ is connected, it either is an even length cycle spanning the vertices of $H$, when $\left|S_{1}\right|=2$, or it contains an even length cycle spanning the vertices of $H$ because of Theorem 1.7 when $|S|>2$. Because $X$ is connected, $S_{2} \neq \emptyset$ so that there is a perfect matching $M$ between $X_{1}$ and $X_{2}$. The spanning cycle of $X_{1}$, together with its obvious copy in $X_{2}$, then join the perfect matching edges $M$ according to the hypotheses of Lemma 2.4. The graph $X$ is then Hamilton-connected or Hamilton-laceable, as required, by Lemma 2.4 .

When $X_{1}$ is not connected, let $\bar{X}$ be the quotient graph obtained by shrinking each component of $X_{1}$ and $X_{2}$ to single vertices, where two vertices in $\bar{X}$ are adjacent if and only if there is an edge between vertices in the two corresponding components.

The quotient graph $\bar{X}$ is a connected Cayley graph on a generalized dihedral group, although its order may not be a multiple of 4 . Nevertheless, the quotient graph $\bar{X}$ has a Hamilton path by Lemma 2.9.

Each edge in $\bar{X}$ corresponds to a perfect matching between the corresponding cycles in $X$ by Lemma 2.2. Thus, the Hamilton path in $\bar{X}$ corresponds to a spanning subgraph of $X$ that is isomorphic to $P_{n} \square C_{m}$. When $m$ is odd, this spanning subgraph is Hamiltonconnected by Lemma 2.7. Thus, $X$ also is Hamilton-connected.

When $m$ is even, the spanning subgraph is Hamilton-laceable by the same lemma. If $X$ is bipartite, then it is Hamilton-laceable and we are done. If $X$ is not bipartite, then we have a little more work to do.

One reason that $X$ may not be bipartite, is that the components of $X_{1}$ and $X_{2}$ are not bipartite. Because $m$ is even and the components of $X_{1}$ and $X_{2}$ are not bipartite, the valency of the vertices in the components has to be at least 3 . Theorem 1.7 then implies the components are Hamilton-connected. It follows that $X$ is Hamilton-connected by Lemma 2.5 .

Finally, suppose the components are bipartite but $X$ is not bipartite. Consider the spanning subgraph $P_{n} \square C_{m}$. We have that $m$ is even and $m>2$ because $\left|S_{1}\right| \geqq 2$. We have that 
$n$ is even and $n \geqq 4$ because each of $X_{1}$ and $X_{2}$ is not connected and the two have the same number of components. The spanning subgraph $P_{n} \square C_{m}$ uniquely determines a bipartition of the vertices of $X$. Because the components themselves are bipartite, there are no edges generated by elements of $S_{1}$ that can violate the bipartition. Thus, there is at least one element of $S_{2}$ whose edges violate the bipartition. In other words, in the notation of Lemma 2.8 , there is an $m$-matching $M(i, k, d)$ whose edges violate the bipartition. This forces both $k$ and $d$ to be odd. It then follows from Lemma 2.8 that $X$ is Hamilton-connected. This completes the proof of Theorem 1.8.

The following two corollaries are immediate consequences of the main theorem and we specifically mention them here because hamiltonicity for Cayley graphs on dihedral groups has been studied with some intensity.

Corollary 4.1. If $X$ is a connected Cayley graph on the dihedral group $D_{n}$, n even, then $X$ has a Hamilton cycle.

Corollary 4.2. Every edge of a connected Cayley graph on a dihedral group $D_{n}$, $n$ even, lies in a Hamilton cycle.

\section{References}

[1] B. Alspach and C.C. Chen, On the hamiltonian laceability of brick products of even cycles, Research Report No. 468, May 1991, Lee Kong Chian Centre for Mathematical Research.

[2] B. Alspach, C. C. Chen and K. McAvaney, On a class of Hamiltonian laceable 3-regular graphs, Discrete Math. 151 (1996), 19-38.

[3] B. Alspach and M. Dean, Honeycomb toroidal graphs are Cayley graphs, Inform. Process. Letters, to appear.

[4] B. Alspach and Y. Qin, Hamilton-connected Cayley graphs on Hamiltonian groups, Europ. J. Combin. 22 (2001), 777-787.

[5] B. Alspach and C. Zhang, Hamilton cycles in cubic Cayley graphs on dihedral groups, Ars Combin. 28 (1989), 101-108.

[6] A. Altshuler, Hamiltonian circuits in some maps on the torus, Discrete Math. 1 (1972), 299314.

[7] C. C. Chen and N. Quimpo, On strongly Hamiltonian Abelian group graphs, in: K. L. Mcavaney (ed.), Combinatorial Mathematics VIII, Lecture Notes in Mathematics 884, Springer, Berlin, 1981, 23-34.

[8] H. Cho and L. Hsu, Ring embedding in faulty honeycomb rectangular torus, Inform. Process. Lett. 84 (2002), 277-284.

[9] H. Cho and L. Hsu, Generalized honeycomb torus, Inform. Process. Lett. 86 (2003), 185-190.

[10] C. Fan, D. R. Lick and J. Liu, Pseudo-Cartesian product and Hamiltonian decompositions of Cayley graphs on Abelian groups, Discrete Math. 158 (1996), 49-62.

[11] D. Marušič and T. Pisanski, Symmetries of hexagonal molecular graphs on the torus, Croatica Chem. Acta 73 (2000), 969-981.

[12] G. M. Megson, X. Liu and X. Yang, Fault-tolerant ring embedding in a honeycomb torus with node failures, Parallel Process. Lett. 9 (1999), 551-561.

[13] G. M. Megson, X. Yang and X. Liu, Honeycomb tori are Hamiltonian, Inform. Process. Lett. 72 (1999), 99-103. 
[14] B. Parhami, A unified formulation of honeycomb and diamond networks, IEEE Trans. Parallel Distrib. Systems 12 (2001), 74-80.

[15] X. Yang, D. J. Evans, H. Lai and G. M. Megson, Generalized honeycomb torus is Hamiltonian, Inform. Process. Lett. 92 (2004), 31-37. 